\title{
Thermodynamic modelling of dynamic cell phenotypes
}

The observed traits, or
phenotypes, of living cell
populations depend on
complex networks of biological
processes. How cells process
information through those
biochemical networks can be
described by the mathematics
of statistical thermodynamics.
Through research at the
National Institute of Standards
and Technology (NIST), a
multidisciplinary team has
clearly shown how the physics
of thermodynamics can
enable an understanding of
the diverse, ever-changing
phenotypes of cells within
populations and provide
predictions of cellular
behaviour. The team's work
could soon bring about new
advances in several cutting-
edge areas of medical research.

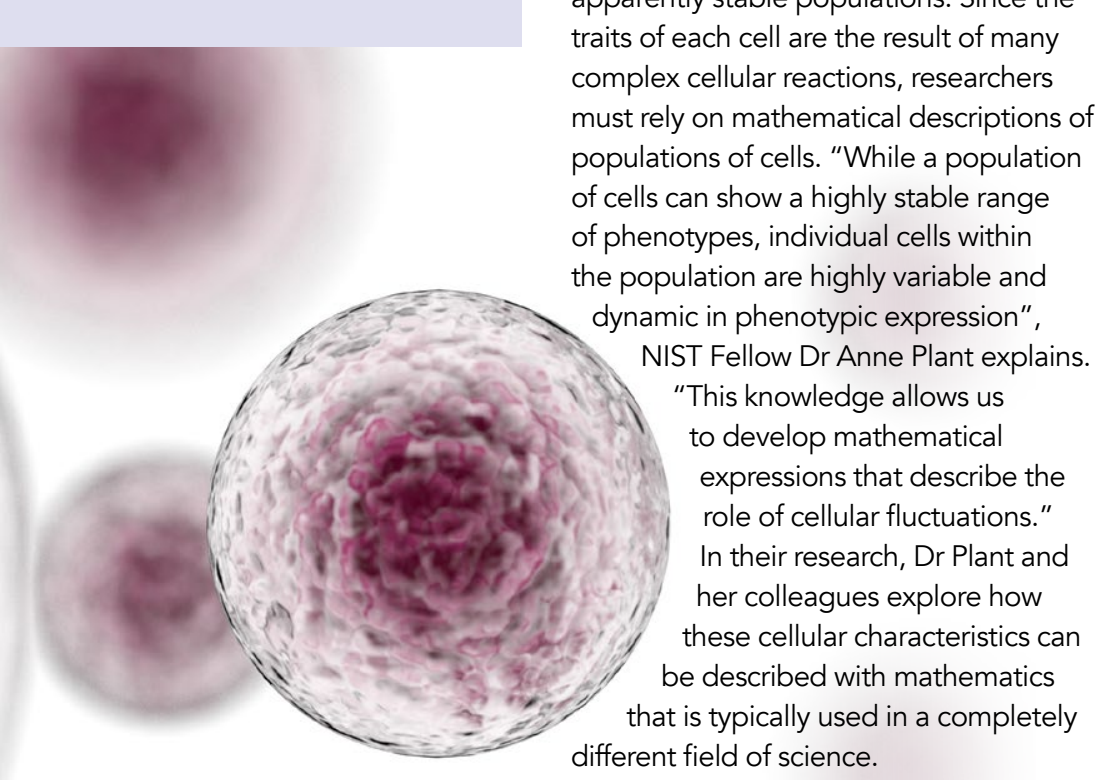
of cancer. Currently, however, many cell-
APPLYING STATISTICAL THERMODYNAMICS TO CELULAR DYNAMICS

ell-based therapies are an
exciting branch of modern medicine. By modifying certain cells taken from a patient or donor, new ways to treat diseases, ranging from based treatments remain in their early stages and must overcome several key challenges before becoming widespread in practice. To gauge how populations of cells will respond as therapies, developers and manufacturers must determine their 'phenotypes' or critical quality attributes, that describe the sets of measurable traits which are expressed by their underlying genetic code.

A major difficulty in doing this lies in the inherent unpredictability of the changes in phenotypes that can occur in individual cells over time, even wirh aits of each cell are the result of many cust rely on mathemalical escrions of ( petween heat mechanical work and the flow of energy within physical systems. At first glance, the field may appear to be of little relevance to biological networks, but the diverse and dynamic nature of individual phenotypes within large, stable cell populations displays a remarkable similarity to the varying physical properties of individual gas molecules in a confined space. To describe these variations, physicists use mathematics first set out by Austrian physicist Ludwig Boltzmann in the 1870s.

According to Boltzmann, the properties of thermodynamic systems relate to the of particles. Crucially a give ensembles result from any one of a vast yet finite range of 'microstates' which describe

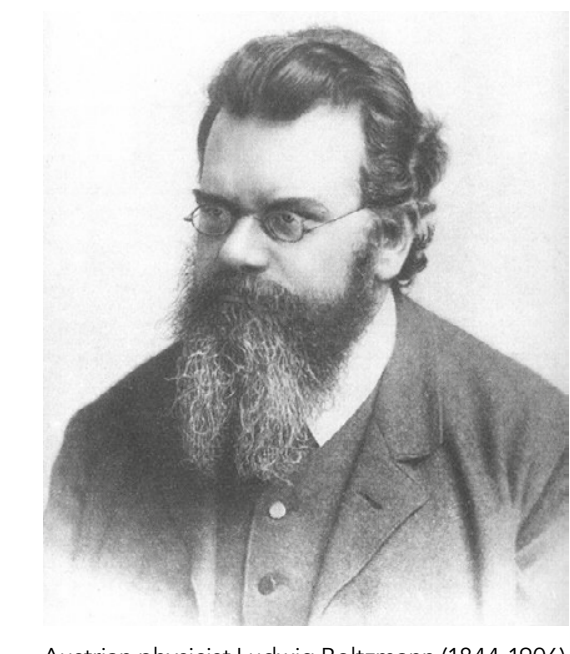

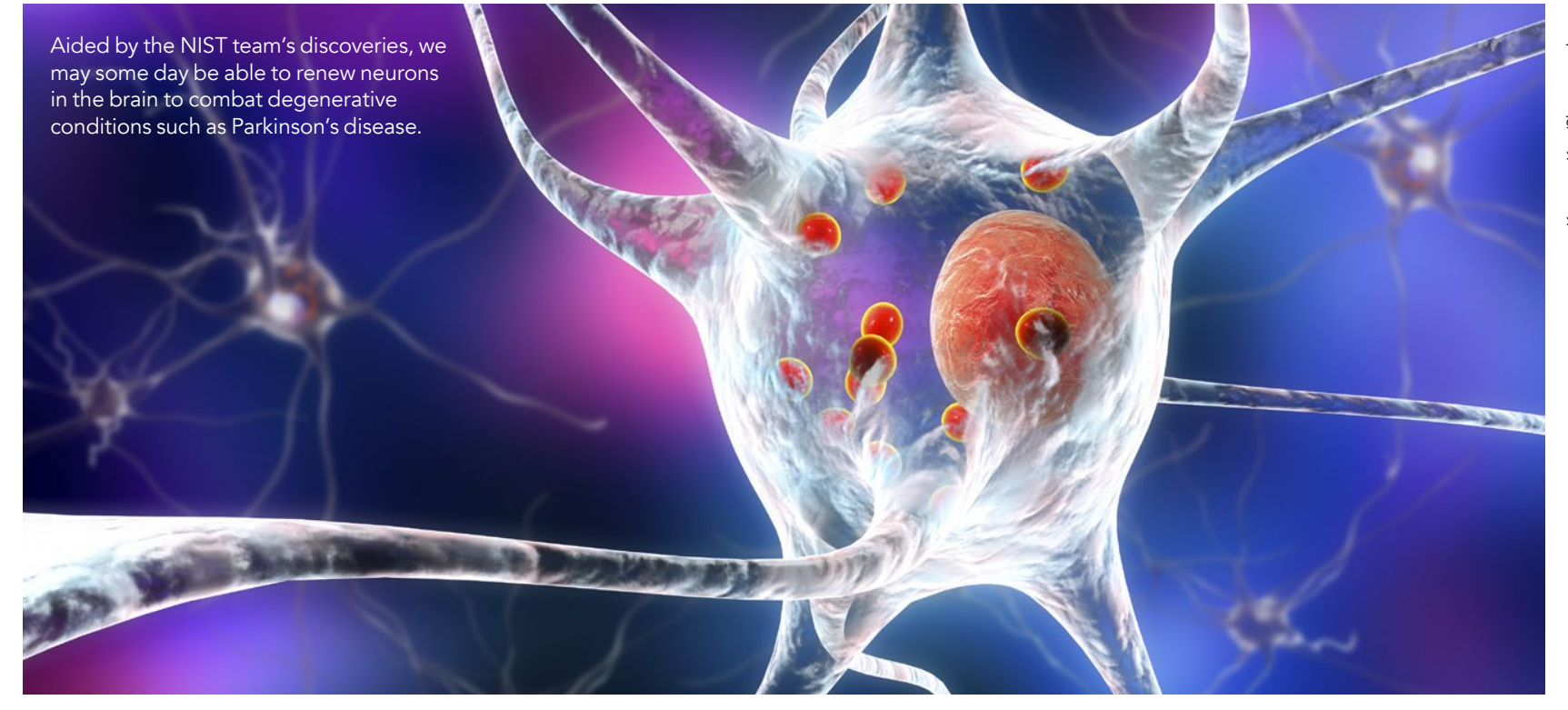

the unique positions and velocities of ach particle in the system. However, energy, a system will not have an eve probability of existing in all microstates across this range. Instead, this probability is defined by a distribution curve or its negative log which is a potential landscape, featuring a peak or valley respectively, at the microstates where the system is most likely to find itself. As biologists later discovered, clear connections can be drawn between the probability distributions of nonequilibrium thermodynamic systems, and those of phenotypes within larg populations of cels.

MEASURING COMPLEXITY AND DER EXPSSION Cell phenpes are of non- positions and welocities individual cells, the expression of genes that control cell phenotypes arise from intersecting networks of different factors, including the rate of synthesis of informationcarrying RNA and reactions involving intricate interactions between protein molecules. Because of this complexity, it is virtually impossible for researchers to quantify all of the details involved in how phenotypes of individual cells come to be, and why individuals are different from one another. Therefore, the NIST team focuses on identifying the most important measuable quantities by essentially folding all the minute,
hard-to-know details of the underlying processes into the overall observations that they can directly measure.

From these results, the researchers th can gain important clues about the within larger groups of cells. Thes sub-populations, or microstates, contain cells with nearly identic phenotypes. "We focus on what can be experimentally measured, such as the rates of fluctuation of lightemitting protein reporters of genes of interest", Dr Plant describes. "These provide mathematical quantities that allow us to predict how fast cells exit from a microstate to populate other

microstates within the population."

To visualise this concept we can think of a lanscape where sub populations

result, cells will find it harder to escap from the sub-populations in attractor inside them. From here, Boltzmann's probability distribution becomes relevant once again, with the more thermodynamically stable microstates being the ones containing more cells. FREE ENERGY

As the team shows, this behaviour is directly comparable to two key aspects of thermodynamics. The first of these is entropy - a term generally used to describe the level of disorder in a macroscale properties of a certain gas: must be in a more disordes particles the probability distributions of nonequilibrium thermodynamic systems, and those of phenotypes within populations of cells.

exist in valleys with varying depths, also called attractor basins. Moreover, the cells' phenotypes are continualy from their attranly tring to escape from their attractor basins. In this microstates with moys stable traits. As a

\section{ENTROPY AND RELATIVE} system. In this context, it relates to the range of microstates that describe the

\section{Connections can be drawn between} changing - they are like jostling
The second is the relative free energy, which is the amount of energy required to keep a system in a steady state, In their latest research, the team
have drawn direct parallels between even when particles are constantly 

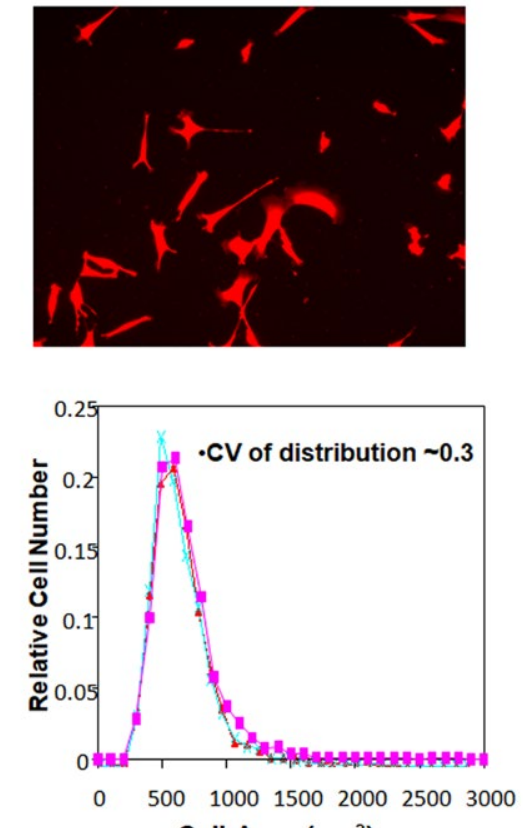

$500 \quad 100015002000$
Cell Area $\left(\mu \mathrm{m}^{2}\right)$
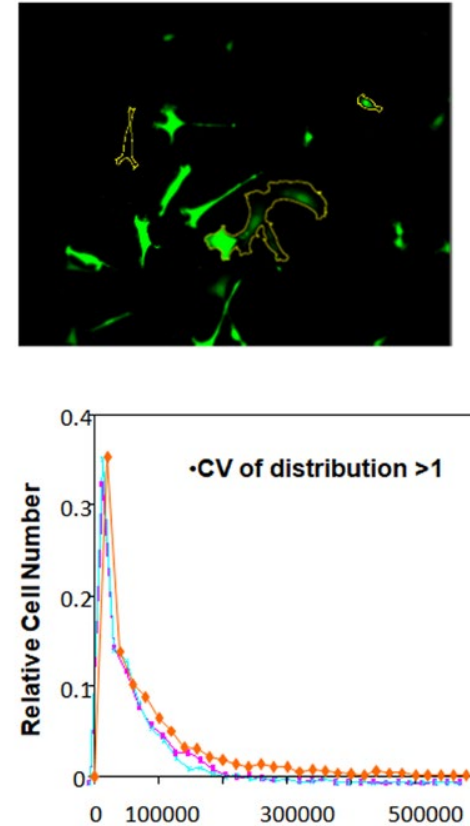

0.100000
Relative GFP Fluorescence

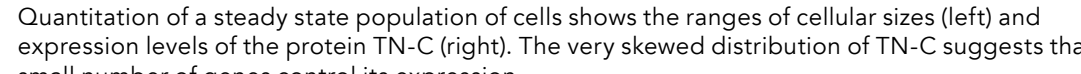

\section{This may improve procedures including} creating T-cells for fighting cancer cells.

each of these quantities, and stable, or 'homeostatic', cell populations - enabling accurate mathematical descriptions of their behaviours. We have shown a direct connection between entropy, relative free energy and the population of steady-state of phenotypes using Boltzmann's mathematics", says Dr Plant. "This analysis allows us to consider the biogred nuin the homeostatic state."

A SMALL NUMBER OF MASTER REGULATOR GENES Graph models are commonly used by systems biologists to map out connections within networks of systems. By modelling the components of a regulated cellular network as 'nodes,' and their connections as 'edges', the low researchers to describe how changes within one node will drive the system connected to it Bas of their new understanding of phenotyp dynamics, the team constructed such a model as a basis for compu researchers could then explore which components must be measured to accurately determine the relative free energy of a real system of cells.

"Simulations revealed that when there are a limited number of genes, or master regulators, that control the network, the distribution of phenotypic responses will be highly asymmetrical", Dr Plant describes. "This suggests that the shape of the steady state distribution of phenotypes in a cell population provides insight into how many network components need to be measured to define the state of the network." Techniques providing a lot finform may be not all that important to the control of the network. The team's new insight now provides an approach that can drastically cut down on the numbers of measurements required to gain the same amount of information. This discovery could have significant implications for future applications of cell populations.

\section{IMPROVING CELL-BASED}

MANUFACTURING

The cells contained in our bodies have the remarkable ability to cure diseases inflicting other parts of the body. The cechnology required to create healthy cells to cure disease is now growing he NIST team's discoveres cold become yet more sophisticated. With a greater knowledge of the dynamic phenotypes of cells in populations, manufacturers and clinicians will be ab to produce and deploy cell therapies far more safely and efficiently.

In turn, this may improve procedures including creating T-cells for fighting cancer cells; surgically applying stem cell-based healthy tissues for repairing damaged tissues; and potentially even renewing neurons in the brain to combat degenerative conditions such as Parkinson's disease. The team continues to explore how their techniques can be advanced even further - bringing these closer to reality.

\section{Behind the Research \\ a \\ E: anne.plant@nist.gov W: https://www.nist.gow \\ people/anne-l-plant
in https://www.linkedin.con
in/anne-plant-06606510/}

\section{Research Objectives}

The NIST group studies the relationship between the mathematics of statistical thermodynamics and the phenotypes of cell populations, paving the way for advances in cell-based therapies.

\section{Detail}

Bio

Dr Anne Plant is a NIST Fellow researching cell imaging and theoretical a Fellow of the AIMBE and AAAS and has previously served as Chief of the Biosystems and Biomaterials Division NIST and at the White House OSTP.

Dr Joseph B. Hubbard's scientific background is in theoretical equilibrium He is currently a NIST Associate collaborating with cell biologists in the Biosystems and Biomaterials Division incorporates concepts and techniques

associated with thermodynamics, information theory via Shannon's and the architecture and signanication, behaviour of gene regulatory networks.

Dr Michael Halter is a research scientist in the Biosystems and Biomaterials Division acience grow seve Cell Systems methods to quantify biological cells. Part of his research is advancing cellular measurement technology, especially microscopic imaging of live pluripotent stem cells. A cricical aspect of his live cel modelling of dynamical processes of cells to develop predictive models.

Dr Swarnavo Sarkar is an associate in at NIST. His current research activivisios include the impact of information acquisition on cellular fitness, information thermodynamics of gene regulatory His previous research has been polymeric and electrochemical systems using stochastic reaction-diffusion and effective-medium methods.

Funding

NIST intramural funding

\section{References}

- Hubbard, J B Halter, M, Sarkar S, Plant A L (2020) The role of fluctuations in determining cellular network thermodynamics. PloS one, 15(3), e0230076. Available at: https://doi.org/10.1371/journal.pone.0230076 - Sarkar, S., Hubbard, J.B., Halter, M., Plant, A.L. (2021). Information Thermodynamics and Reducibility of Large Gene Networks. Entropy, 23(1), 63. Available at: https:// doi.org/10.3390/e23010063

- Sisan, D.R., Halter, M., Hubbard, J.B., Plant, A.L. (2012). Predicting rates of cell state change caused by stochastic fluctuations using a data-driven landscape model. Proceedings of the National Academy of Sciences, 109(47), 19262-19267. Available at: https://do $\mathrm{org} / 10.1073 /$ pnas. 1207544109

Plant, A.L., Halter, M., Stinson, J. (2020). Probing pluripotency gene regulatory networks with quantitative live cell imaging. Computational and Structural https://doi.org/10.1016/j.csbj.2020.09.025

\section{Personal Response}

How readily could your simulations be applied in a real cell-based manufacturing context, such as
production of an immune cell therapy? II One of the greatest challenges in developing cell thesential is knowing what measurable charactefistics are This dilemma is a challenge from the basic research stage to manufacturing. How manufacturing conditions influence product characteristics is important for the affordable. We anticipate our work will aid in knowing what few measurements need to be monitored in order to predict a successfully manufactured product.

\section{NLTT}

National Institute of
Standards and Technology 